\title{
Intratracheal myiasis followed by tracheal- esophageal fistula: report of a rare case and literature review
}

\author{
Huang Wendi ${ }^{\dagger}$, Chao Zeng ${ }^{\dagger}$, Weidong Song ${ }^{*}$ and Ping $\mathrm{Xu}^{*}$ (D)
}

\begin{abstract}
Background: To enhance awareness of the clinical features and prevention of endotracheal myiasis.

Case presentation: A case of intratracheal myiasis is reported. A 61-year-old male patient with a history of laryngectomy was admitted to hospital due to tracheostomal hemorrhage of $3 \mathrm{~h}$ duration. Intratracheal myiasis was confirmed by bronchoscopy, and the patient underwent bronchoscopic intervention, which was complicated by a tracheal-esophageal fistula and resolved by endotracheal stenting. Twenty months after stent placement, the fistula had not healed.

Conclusion: Intratracheal myiasis has serious complications and is difficult to treat. For post-tracheostomy patients, healthcare providers and caregivers should pay attention to the care and monitoring of wounds and maintenance of a tidy, clean living environment to prevent intratracheal myiasis.
\end{abstract}

\section{Background}

Myiasis is the invasion of dipterous insect larvae into tissues. The larvae can invade human skin, gangrenous tissue, and natural cavities. Myiasis of the trachea is very rare with only a few cases previously reported. Furthermore, intratracheal myiasis followed by a trachealesophageal fistula has not, to the best of our knowledge, been reported previously [1-5].

\section{Case presentation}

A 61-year-old man from Shenzhen presented with tracheostomal hemorrhage of $3 \mathrm{~h}$ duration. One year previously, the patient had been diagnosed with laryngeal squamous cell carcinoma by electronic laryngoscopy, and had undergone partial laryngectomy and tracheotomy under general anesthesia. Following these procedures, he was discharged and recovered well. The tracheotomy was maintained after discharge. The patient and his family cleaned the tracheal cannula themselves which may have resulted in inadequate care. The patient

\footnotetext{
* Correspondence: Songwd123@hotmail.com; ping-xu@hotmail.com 'Wendi Huang and Chao Zeng contributed equally to this work. Departments of Respiratory and Critical Care Medicine, Peking University Shenzhen Hospital, Lianhua Road, Shenzhen 518036, Guangdong, China
}

visited our hospital due to tracheostomal hemorrhage of $3 \mathrm{~h}$ duration.

Physical examination at admission showed peritracheostomal redness and correct positioning of the tracheal cannula. Bloody secretions were seen in the dressing. Electronic laryngoscopy showed postoperative changes in the glottic area, with scar formation; the airway was patent. The results of a complete blood count were $9.58 \times 10^{9} / \mathrm{L}$ white blood cells, $74.4 \%$ neutrophils, and $1.0 \%$ eosinophils. After admission, the tracheal cannula was removed, and the surface of the cannula was dirty. The stomal tissue was red and swollen. On the day after removal of the tracheal cannula, the patient coughed out maggot larvae through the tracheostomy, and several larvae were found in the peri-stomal area.

Computed tomography of the chest showed a hyperdense mass involving the trachea (Fig. 1a, arrow). The peri-esophageal and peri-tracheal fat spaces were blurred (Fig. 1b, arrow). Bronchoscopy confirmed the presence of live larvae in the left lower tracheal segment, with fibrous granulation and tracheal stenosis. Furthermore, heavy granulation was found in the upper and middle thirds of the trachea. A tracheostenosis with a smallest diameter of about $0.6 \mathrm{~cm}$ was noted (Fig. 1c); it was resolved using electrocautery to burn the larvae (Fig. 1d), 


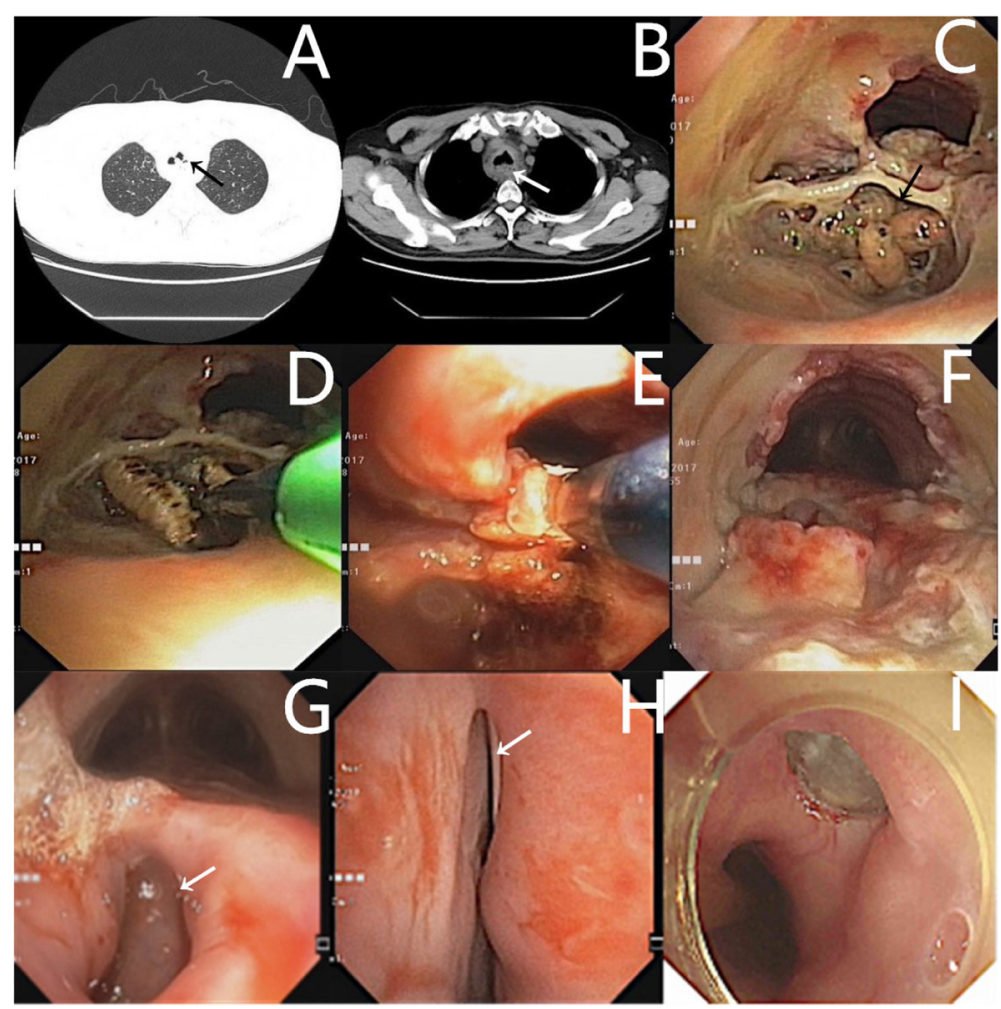

Fig. 1 a, b A hyperdense mass involving the trachea (black arrow) and blurred fat space around the oesophagus and trachea (white arrow). c The first fibre-optic bronchoscopy showed a larval burrow with live larvae (black arrow) in the lower trachea, and tracheal stenosis. $\mathbf{d}$ The tracheostenosis was resolved by using electrocautery to burn the larvae, and making linear and circular incisions into the larval burrow. e The larvae were removed using cryotherapy and forceps, and the basal granulation was cleared. $\mathbf{f}$ The second fibre-optic bronchoscopy revealed granulation and necrosis covering the trachea, with no burrow or live larvae. The tracheal stenosis had improved. $\mathbf{g}, \mathbf{h}$ The third fibre-optic bronchoscopy showed a tracheal-oesophageal fistula in the lower trachea (white arrow), and the indwelling gastric tube under the fistula (white arrow). i Follow-up gastroscopy performed 12 months after the procedure showed incomplete healing, with the tracheal-oesophageal fistula covering the stent

and by making linear and circular incisions in the larval burrow and removing the larvae using cryotherapy and forceps (Fig. 1e) and clearing the basal granulation. This procedure resulted in improvement of the tracheal stenosis. Bronchoscopy showed that the left and right main bronchi and their upper and lower segments were patent. One week later, follow-up fiberoptic bronchoscopy showed granulation and membranous necrosis in the upper and middle trachea. The larval burrow was absent from the left lower trachea, but the site was covered with granulation and necrotic tissue. The trachea was restored to three-quarters of its normal size. We removed the necrotic tissue and granulation using forceps and freezing (Fig. 1f).

After the procedure, the patient experienced cough after taking food. Follow-up bronchoscopy showed a trachealesophageal fistula of about $1.5 \mathrm{~cm}$ (Fig. $1 \mathrm{~g}$ and h) in the lower trachea, $2 \mathrm{~cm}$ above the carina, so an indwelling gastric tube was placed which was visible on bronchoscopy passing through the tracheoesophageal fistula. The thoracic surgeon recommended conservative medical treatment, However, the patient continued to cough up food so bronchoscopy was performed 1 month later which showed that the tracheal-esophageal fistula had not properly healed. Next, a Y-type tracheal silicone stent was placed. The patient's condition has been stable since that time. Follow-up gastroscopy performed 12 months after the procedure showed that the tracheal-esophageal fistula had not healed and covered the Y-type tracheal silicone stent (Fig. 1i), for which we suggested surgical repair.

\section{Discussion and conclusions}

In this case, intratracheal myiasis resulted from invasion of the trachea by dipterous larvae. Few cases of bronchial myiasis have been reported [1]. A search of PubMed using the keywords 'human intratracheal myiasis' or 'tracheopulmonary myiasis,' or 'bronchial myiasis' revealed five reports of five cases published up to July 2018 (Table 1). The cases were sporadic, without age or sex predilection, and all occurred in tropical and subtropical regions where flies are prevalent following endotracheal intubation or tracheotomy [3-7]. 
Table 1 Reported cases of intratracheal myiasis

\begin{tabular}{lllllll}
\hline Authors and year & Patient sex/age & Country & Concomitant diseases & Involved areas & Confirmative evidence & Clinical manifestations \\
\hline Cornet et al. 2002 & Female/60 years & US & No & Intratracheal & Cough out & Cough, haemoptysis \\
Cecchini et al. 2012 & Male/47 years & France & Diabetes, septic shock & Left main bronchus & Bronchoscopy & No \\
Arindom et al. 2015 & Male/13 years & Oman & No & Right upper bronchus & Bronchoscopy & Cough, dyspnoea \\
Ahadizadeh et al. 2015 & Male/53 years & US & Cardiac arrest & Intratracheal & Inside tracheal catheter & No \\
Ince et al. 2015 & Female/8 months & Turkey & N/A & Intratracheal & N/A & N/A \\
\hline
\end{tabular}

N/A not accessible

Notably, the case reported here is the only case with clear evidence of intratracheal colonization; furthermore, intratracheal myiasis followed by tracheal-esophageal fistula has not, to the best of our knowledge, been reported previously. The patient in this case underwent tracheotomy for nasopharyngeal carcinoma. The invasion was due to a female fly laying eggs in the main trachea through the tracheotomy. Tracheostomal myiasis has also been reported outside of China [8-10]. The open space formed by the tracheotomy and the odor emitted by the secretions at the fistula are important contributing factors. In addition, poor personal hygiene and the postoperatively suppressed cough reflex provide favorable conditions for intratracheal colonization. The treatment of myiasis involves surgical removal of larvae and administration of the broad-spectrum antiparasitic drug ivermectin [4]. For the current case, surgical treatment was feared to be too traumatic in the face of severe airway stenosis, so fiberoptic bronchoscopic treatment was initially attempted. The larvae were carefully removed from the surface of the burrow using tracheoscope forceps, taking care not to drop them into the lower airway; unfortunately, more maggots hatched and crawled out afterwards. Bronchoscopy revealed different sizes of maggots in the airway including $0.1 \mathrm{~mm}$ living and peristaltic larvae in a tracheal tissue sample. To completely remove larvae and reduce the risk of local scar formation leading to tracheal stenosis, electrocautery and cryotherapy were performed. Follow-up bronchoscopy was used to assess recovery and treatment complications (such as scar re-stenosis and tracheal-esophageal fistula). Postoperatively, the patient continued to cough up food. A tracheal-esophageal fistula was observed by fiber-optic bronchoscopy, and was considered a result of larvae damaging the tracheal mucosa. Medical treatment was considered, given that the patient was in poor physical condition and probably intolerant of surgery. The diameter of the fistula was $1.5 \mathrm{~cm}$; biogel therapy and chemical cauterization for fistulas of this size often fails [11, 12]. Therefore, airway and/or esophageal stenting was considered. Shin et al [13] reviewed stenting methods for tracheal-esophageal fistulae of various causes and types, which consist of: (1) esophageal stenting for trachealesophageal fistulae combined with severe esophageal stricture but very mild, if any, tracheal stenosis; (2) tracheal stenting when the esophagus and trachea are without remarkable stenosis; (3) tracheal stenting for severe tracheal stenosis without esophageal stenosis; and (4) esophageal and tracheal stenting for concomitant severe esophageal and tracheal stenosis. As the patient described here had no significant esophageal or tracheal stenosis, coated graft stenting was preferred. Finally, bronchoscopic tracheal stenting and indwelling gastric catheterization were performed. At 20 months post-op, the fistula had not healed and the stent remained to prevent reflux.

In this patient, the maggots were located at the junction between the lower end of the tracheal cannula and the trachea. We speculate that the maggots had been present for several weeks and had gnawed through the tracheal and esophageal walls, resulting in tracheostomal hemorrhage. The maggots climbed up to the tracheal wound after the tracheal cannula was removed. If not properly treated in a timely manner, intratracheal myiasis may cause severe complications, including secondary infection, respiratory failure [5], tracheal stenosis, and tracheal-esophageal fistula. The prognosis is generally good when no complication occurs. Prevention is feasible, particularly posttracheostomy, in older patients of low socio-economic status, those living in unsanitary environments, those who abuse alcohol, patients with central nervous system conditions, and those with poor personal hygiene [14].

In conclusion, tracheal-esophageal fistula is a serious complication of endotracheal myiasis and is difficult to treat. Tracheal stenting can improve the resulting reflux but cannot treat the condition. For post-tracheostomy patients, healthcare providers and caregivers should pay attention to the care and monitoring of wounds and maintenance of a tidy, clean living environment to prevent intratracheal myiasis.

\section{Abbreviations \\ cm: centimetre; g: gramma; L: Liter \\ Acknowledgments \\ The authors are indebted to the nurses of the bronchoscopy Room for making contributions to this study.}

Authors' contributions

Data acquisition, analysis and interpretation: PX, WDH, CZ, WSD, Writing the article or substantial involvement in its revision: WDH, PX. All authors have read and approved the manuscript, and ensure that this is the case. 
Funding

None.

\section{Availability of data and materials}

The materials described in the manuscript are available from the corresponding author on reasonable request.

\section{Ethics approval and consent to participate}

The study was approved by the Ethics Committee of Peking university Shenzhen Hospital. Informed written consent was obtained from the patient for publication of this case report and accompanying images.

\section{Consent for publication}

All presentations of case reports have consent to publish, Informed consent for publication of mages or other personal or clinical details was obtained from the patient.

\section{Competing interests}

The authors declare that they have no competing interests.

Received: 2 June 2019 Accepted: 29 November 2019

Published online: 17 December 2019

\section{References}

1. Cornet M, Florent M, Lefebvre A, Wertheimer C, Perez-Eid C, Bangs MJ, et al. Tracheopulmonary myiasis caused by a mature third-instar Cuterebra larva: case report and review. J Clin Microbiol. 2003;41:5810-2.

2. Cecchini J, Prost ND, Mekontso-Dessap A, et al. Life-threatening endobronchial myiasis. Intensive Care Med. 2012;38(10):1727-8.

3. Aich A, Alismaili S, Ramadhan FA, et al. Eosinophilic pneumonia in a patient with bronchial Myiasis: case report and literature review. Sultan Qaboos Univ Med J. 2015;15(4):e546-9.

4. Ahadizadeh EN, Ketchum HR, Wheeler R. Human cutaneous Myiasis by the Australian sheep blowfly, Lucilia cuprina (Diptera: Calliphoridae), in Oklahoma. J Forensic Sci. 2015;60(4):1099-100.

5. Ince $E$, Oğuzkurt P, Gezer HÖ, et al. Aspiration of an interesting foreign body: Myiasis. Turk J Pediatr. 2015;57(6):621-3.

6. Cao XL, Sang YH, Yang YL, et al. Overview of 291 cases of human myiasis in China from 2003 to 2013. Guide Chinese Med. 2015;13(8):37-9.

7. Francesconi F, Lupi O. Myiasis. Clin Microbiol Rev. 2012;25:79-105.

8. Franza R, Leo L, Minerva T, et al. Myiasis of the tracheostomy wound: case report. Acta Otorhinolaryngol Ital. 2006;26(4):222-4.

9. Batista-da-Silva JA, Borja GE, Queiroz MM. Patient with tracheostomy parasitized in hospital by larvae of the screwworm, Cochliomyia hominivorax. J Insect Sci. 2011;11(163):163.

10. Severini F, Nocita E, Tosini F. Myiasis of the tracheostomy wound caused by Sarcophaga (Liopygia) argyrostoma (Diptera: Sarcophagidae): molecular identification based on the mitochondrial cytochrome c oxidase I gene. J Med Entomol. 2015;52(6):123-30.

11. Mehta HJ, Malhotra P, Begnaud A, et al. Treatment of alveolarpleural fistula with endobronchial application of synthetic hydrogel. Chest. 2015;147(3):695-9.

12. Stratakos G, Zuccatosta L, Porfyridis I, et al. Silver nitrate through flexble bronchoscope in the treatment of bronchopleural fistulae. J Thorac Cardiovasc Surg. 2009;138(3):603-7.

13. Shin JH, Kim JH, Song HY. Interventional management of esophagorespiratory fistula. Korean J Radiol. 2010;11(2):133-40.

14. Failocrojas VE, Silvadíaz H. Review of cases and a patient report of Myiasis with tracheostomy, Peru. Emerg Infect Dis. 2016;22(3):563-5.

\section{Publisher's Note}

Springer Nature remains neutral with regard to jurisdictional claims in published maps and institutional affiliations.

Ready to submit your research? Choose BMC and benefit from:
- fast, convenient online submission
- thorough peer review by experienced researchers in your field
- rapid publication on acceptance
- support for research data, including large and complex data types
- gold Open Access which fosters wider collaboration and increased citations
- maximum visibility for your research: over 100M website views per year
At BMC, research is always in progress.
Learn more biomedcentral.com/submissions

